\title{
Random plasma glucose in early pregnancy is a better predictor of gestational diabetes diagnosis than maternal obesity
}

\author{
Claire L. Meek ${ }^{1,2,3}$ • Helen R. Murphy ${ }^{1,2,4}$ • David Simmons ${ }^{2,5}$
}

Received: 18 August 2015 / Accepted: 23 October 2015 / Published online: 20 November 2015

(C) The Author(s) 2015. This article is published with open access at Springerlink.com

\begin{abstract}
Aims/hypothesis Asymptomatic pregnant women are screened for gestational diabetes (GDM) at 24-28 weeks' gestation. Recent guidelines also recommend screening early in gestation to identify undiagnosed pre-existing overt diabetes. We assessed the performance of random plasma glucose (RPG) testing at antenatal booking in predicting GDM diagnosis later in pregnancy.

Methods Data from 25,543 consecutive singleton pregnancies at the Rosie Hospital in Cambridge (UK) were obtained from hospital electronic records as a service evaluation. All women were invited for an antenatal RPG (12-16 weeks) and a $50 \mathrm{~g}$ glucose challenge test (GCT; 24-28 weeks) with a 75 g OGTT if GCT $>7.7 \mathrm{mmol} / 1(139 \mathrm{mg} / \mathrm{dl})$.

Results At booking, 17,736 women had an RPG that was able to predict GDM (receiver operating characteristic AUC 0.8)
\end{abstract}

Electronic supplementary material The online version of this article (doi:10.1007/s00125-015-3811-5) contains peer-reviewed but unedited supplementary material, which is available to authorised users.

Claire L. Meek

clm70@cam.ac.uk

1 The Wellcome Trust-MRC Institute of Metabolic Science, Metabolic Research Laboratories, University of Cambridge, Addenbrooke's Hospital, Box 289, Hills Road, Cambridge CB2 0QQ, UK

2 Wolfson Diabetes and Endocrinology Clinic, Cambridge University Hospitals, Addenbrooke's Hospital, Cambridge, UK

3 Department of Clinical Biochemistry, Cambridge University Hospitals, Addenbrooke's Hospital, Cambridge, UK

4 Department of Medicine, University of East Anglia, Norwich Medical School, Norwich, UK

5 School of Medicine, University of Western Sydney, Campbelltown, NSW, Australia according to various diagnostic criteria in common use. A cutoff point of $\geq 7.5 \mathrm{mmol} / 1(135 \mathrm{mg} / \mathrm{dl})$ gave a sensitivity of 0.70 and a specificity of 0.90 for GDM diagnosis. Theoretically, using this screening policy, $13.2 \%$ of women would have been categorised at high risk (26.3\% had GDM) and $86.8 \%$ of women at low risk ( $1.7 \%$ had GDM). RPG performed better than maternal age (AUC 0.60) or BMI (AUC 0.65) at predicting GDM diagnosis.

Conclusions/interpretation RPG at booking has reasonable performance as a screening test and is better than maternal age or BMI for identifying women at high risk of GDM. RPG cannot replace OGTT for diagnosis but it may be useful to exclude women who do not need further investigation for GDM and to identify women who could be prioritised for early diagnosis or lifestyle interventions.

$\begin{array}{ll}\begin{array}{l}\text { Abbreviations } \\ \text { ACOG }\end{array} & \begin{array}{l}\text { American Congress of Obstetricians and } \\ \text { Gynecologists }\end{array} \\ \text { FPG } & \text { Fasting plasma glucose } \\ \text { GDM } & \text { Gestational diabetes } \\ \text { GCT } & \text { Glucose challenge test } \\ \text { IADPSG } & \begin{array}{l}\text { International Association of the Diabetes in } \\ \text { Pregnancy Study Groups }\end{array} \\ \text { LGA } & \text { Large for gestational age } \\ \text { NICE } & \text { National Institute for Health and Care Excellence } \\ \text { RPG } & \text { Random plasma glucose } \\ \text { USPSTF } & \text { US Preventative Services Task Force }\end{array}$

\section{Introduction}

Gestational diabetes (GDM), defined as carbohydrate intolerance causing hyperglycaemia with first onset or recognition 
during pregnancy, is associated with adverse maternal and fetal outcomes $[1,2]$. The Hyperglycaemia and Adverse Perinatal Outcomes (HAPO) study identified that all degrees of hyperglycaemia are linked linearly to adverse outcomes in pregnancy, with no obvious inflection point for this risk [3]. This has led to considerable difficulty in defining GDM. The International Association of the Diabetes in Pregnancy Study Groups (IADPSG) recommended setting diagnostic cut-off points at a level consistent with an OR of 1.75 [4] (see electronic supplementary material [ESM] Table 1), which has resulted in a larger number of diagnoses. These criteria have been adopted by the WHO [5] and the ADA [6] but have not been universally accepted [7, 8], in part due to concerns about resource allocation with the increasing prevalence of GDM and concerns about excessive medicalisation of healthy pregnancy [9]. Although many countries are adopting the WHO 2013 criteria, there remains great heterogeneity of diagnostic criteria used for GDM, even within the same country [10].

There is also considerable controversy about how best to identify women with GDM. The ADA and US Preventive Services Task Force (USPSTF) recommend that all pregnant women should be screened at 24-28 weeks unless they are known to have pre-existing diabetes [6]. The American Congress of Obstetricians and Gynecologists (ACOG) guidelines agree that all women should be screened at 24-28 weeks' gestation but suggest that this could be performed by assessment of 'the patient's medical history, clinical risk factors, or laboratory screening test results to determine blood glucose levels' [11]. Guidelines published by the National Institute for Health and Care Excellence (NICE) in the UK recommend screening only women with risk factors, including obesity, previous GDM, family history of diabetes or ethnicity with a high diabetes prevalence [8]. Although universal screening policies whereby all women are screened biochemically for GDM are considered expensive, there is some concern that risk-factor-based approaches miss many cases who might otherwise benefit from treatment [12] and create added complexity for healthcare professionals conducting the screening [13].

There is also considerable controversy regarding the type and timing of blood tests with which to diagnose GDM. The ACOG and USPSTF recommendations from the USA have favoured a two-step approach using a $50 \mathrm{~g}$ glucose challenge test (GCT) and a confirmatory test using the $100 \mathrm{~g}$ OGTT [11]. The WHO, ADA and IADPSG all recommend a onestep approach, using a $75 \mathrm{~g}$ OGTT with glucose determination carried out at baseline and at 1 and $2 \mathrm{~h}$ after the glucose load. The NICE guidelines recommend a $75 \mathrm{~g}$ OGTT with glucose measurement at baseline and at $2 \mathrm{~h}$ post load. Other groups have suggested that tests such as fasting plasma glucose [14] or random plasma glucose (RPG) [15] might have validity in screening for GDM, either alone or as a method of rationing OGTTs. A previous systematic review of the use of RPG to screen for GDM concluded that there was inadequate evidence to support the use of RPG, but only six relevant studies (including a total of 3,537 women) were identified [16].

In our institution, an RPG is taken at antenatal booking (12-16 weeks) to exclude overt diabetes [17]. Because women who develop GDM have abnormal glucose handling or insulin resistance prior to pregnancy, we hypothesised that the RPG may also be able to identify women who may later develop GDM. The aim of this study was to assess the usefulness of an RPG taken at antenatal booking as a screening test for GDM, diagnosed at any point during pregnancy.

\section{Methods}

Population and standard care As described previously [18], data from all singleton pregnancies (2004-2008) at the Rosie Hospital, Cambridge Universities NHS Foundation Trust were obtained retrospectively from hospital medical and obstetric records as part of an approved service evaluation. At that time in our institution, all pregnant women had been invited to attend an antenatal appointment at which RPG $(n=17$, 736; typically $12-16$ weeks' gestation) was measured. Women with RPG $>7.0 \mathrm{mmol} / 1$ or who had a previous diagnosis of GDM were offered an early $75 \mathrm{~g}$ OGTT. All women without known GDM/pre-existing diabetes were screened at 24 28 weeks with a 50 g GCT: women with a GCT result $>7.7 \mathrm{mmol} / \mathrm{l}(139 \mathrm{mg} / \mathrm{dl})$ were then referred for a $75 \mathrm{~g}$ OGTT [18]. Additional OGTTs were performed in later pregnancy if symptoms were present. Therefore, all women who had an OGTT $(n=3,848)$ had already had at least one abnormal glucose test result during pregnancy, symptoms consistent with hyperglycaemia or GDM in a previous pregnancy. Women with known pre-existing diabetes were excluded from the study. The study period of 2004-2008 was chosen as electronic records, screening procedures and treatment protocols were constant during this time.

Laboratory analysis Both venous and capillary blood samples were used during 2004-2008 for glucose testing in our institution. Venous blood was collected using fluoride-oxalate tubes and analysed using a hexokinase method (Dimension RXL MAX Clinical Chemistry System; Siemens Healthcare Diagnostics, Deerfield, IL, USA) in our accredited laboratory (Clinical Pathology Accreditation, UK Accreditation Service, Feltham, UK). Capillary samples were analysed using the Bayer Elite glucose monitoring system (Bayer, Newbury, UK). Although both laboratory and point-of-care methods were regularly calibrated, small differences exist between capillary and venous glucose testing [19]. The same diagnostic criteria were used for both capillary and venous tests. 
Statistical analysis Receiver operating characteristic (ROC) curves were used to estimate AUC and the $95 \%$ CI. Statistical analysis was performed using STATA (version 12.0; StataCorp, College Station, TX, USA).

\section{Results}

Records were obtained for 25,789 births; 25,543 records were included in the analysis after exclusion of pregnancies resulting in miscarriage $(n=59)$ or termination $(n=65)$ and records with no birthweight information $(n=3)$, duplicate data $(n=20)$ and data consistent with overt diabetes (RPG $\geq 11.1 \mathrm{mmol} / 1$ at booking; $n=99$ ). Of these, only 17,736 pregnancies had a documented RPG at booking. Those without RPG measurements recorded have been described more thoroughly elsewhere [17].

Baseline characteristics of women are described according to the presence or absence of GDM according to the IADPSG criteria (Table 1). As expected, women with GDM present had higher rates of obesity (BMI $\geq 30 \mathrm{~kg} / \mathrm{m}^{2}$ ), higher age at delivery and were more likely to give birth to a macrosomic or large-for-gestational-age (LGA) infant compared with women who did not have GDM.

The ability of the RPG to predict GDM was tested using ROC curves (Fig. 1). RPG was able to predict GDM according to the IADPSG ( $n=1,181$ positive diagnoses, $n=884$ with RPG; AUC 0.81; 95\% CI 0.80, 0.83), NICE 2015 ( $n=1,055$ positive diagnoses, $n=806$ with RPG; AUC $0.81 ; 95 \%$ CI $0.79,0.83)$, WHO 1999 ( $n=1,016$ positive diagnoses; $n=775$ with RPG; AUC $0.81 ; 95 \%$ CI $0.79,0.83)$ and Modified WHO 1999 ( $n=1,025$ positive diagnoses; $n=782$ with RPG; AUC 0.80; 95\% CI 0.78, 0.83) criteria.
Using a cut-off value of RPG $\geq 7.5 \mathrm{mmol} / \mathrm{l}(135 \mathrm{mg} / \mathrm{dl})$, which produced best overall performance of sensitivity and specificity, RPG was able to predict GDM diagnosis using IADPSG (sensitivity 0.70, specificity 0.90), NICE 2015 (sensitivity 0.69, specificity 0.89), WHO 1999 (sensitivity 0.69, specificity 0.89) and Modified WHO 1999 (sensitivity 0.69 , specificity 0.89 ) criteria. In this dataset of 17,736 pregnancies with RPG data, 15,396 women (86.81\%) fell below this threshold and 2,340 fell above the threshold (13.19\%).

As the clinical value of RPG would be in excluding women who do not need further investigation for GDM, a higher cutoff value of $\geq 8.5 \mathrm{mmol} / 1(153 \mathrm{mg} / \mathrm{dl})$ was also assessed to maximise specificity while providing acceptable sensitivity. At this level, RPG was able to predict GDM according to IADPSG (sensitivity 0.43, specificity 0.97), NICE 2015 (sensitivity 0.42, specificity 0.96), WHO 1999 (sensitivity 0.42, specificity 0.96) and Modified WHO 1999 (sensitivity 0.42 , specificity 0.96 ) criteria. In this dataset of 17,736 pregnancies with recorded RPG, 16,789 women fell below this threshold and 947 fell above the threshold.

As the range of RPG values was considerable in women who later developed GDM (see Fig. 1e), a cut-off point of around $\geq 4.7 \mathrm{mmol} / 1(85 \mathrm{mg} / \mathrm{dl})$ was required to give a sensitivity of $90 \%$ using any diagnostic criteria. Of the 3,863 women who had values $<4.7 \mathrm{mmol} / \mathrm{l}, 68(1.76 \%)$ were eventually diagnosed with GDM according to IADPSG criteria.

Theoretically, adopting an RPG screening policy in this population using IADPSG criteria with a cut-off point of $\geq 7.5 \mathrm{mmol} / \mathrm{l}(135 \mathrm{mg} / \mathrm{dl})$ would have identified 2,340 women as being at high risk of GDM (Fig. 2; 615 [26.3\%] were later found to be positive for GDM). This screening policy would also have identified 15,396 women as being at low risk of GDM (of whom 15,127 [98.3\%] were negative for GDM).
Table 1 Characteristics of all pregnancies and those identified as GDM-positive and GDM-negative according to the IADPSG criteria

\begin{tabular}{|c|c|c|c|}
\hline Characteristic & All pregnancies & $\begin{array}{l}\text { GDM-negative } \\
\text { (IADPSG) }\end{array}$ & $\begin{array}{l}\text { GDM-positive } \\
\text { (IADPSG) }\end{array}$ \\
\hline No. of pregnancies & 25,543 & 24,362 & 1,181 \\
\hline Maternal age $\geq 30$ years at delivery & $15,773(61.8)$ & $14,890(61.1)$ & $883(74.8)$ \\
\hline Maternal smoking at booking & $2,416(9.5)$ & $2,342(9.6)$ & $74(6.3)$ \\
\hline Maternal white ethnicity & $22,762(89.3)$ & $21,785(89.6)$ & $977(82.8)$ \\
\hline Maternal obesity & $3,016(13.9)$ & $2,701(13.1)$ & $315(30.0)$ \\
\hline Primiparous & $9,895(38.8)$ & $9,437(38.8)$ & 458 (38.9) \\
\hline Macrosomia (BW >4 kg) & $3,097(12.1)$ & $2,854(11.7)$ & $243(20.6)$ \\
\hline LGA (BW >90th percentile) & $3,010(12.2)$ & $2,700(11.5)$ & $310(26.9)$ \\
\hline \multicolumn{4}{|l|}{ Method of delivery } \\
\hline SVD & $15,321(60.0)$ & $14,790(60.7)$ & $531(45.0)$ \\
\hline $\mathrm{CS}$ & $6,795(26.6)$ & $6,301(25.9)$ & $494(41.8)$ \\
\hline
\end{tabular}

Data are shown as $n(\%)$

Note that approximately $99.9 \%$ of records had data available for pregnancy outcome, mode of delivery and antenatal complications but only $84.9 \%$ of records had data available for their usual maternal adult BMI

BW, birthweight; CS, Caesarean section; SVD, spontaneous vertex delivery 
Fig. 1 (a-d) ROC curves for the use of RPG to predict GDM diagnosed using the IADPSG criteria (a), the NICE 2015 criteria (b), the WHO 1999 (WHO-99) criteria (c) and the modified (Mod) WHO 1999 criteria (d); AUC and 95\% CIs are also shown. The distribution of RPG values in women who were negative (e) and positive (f) for GDM according to the IADPSG criteria
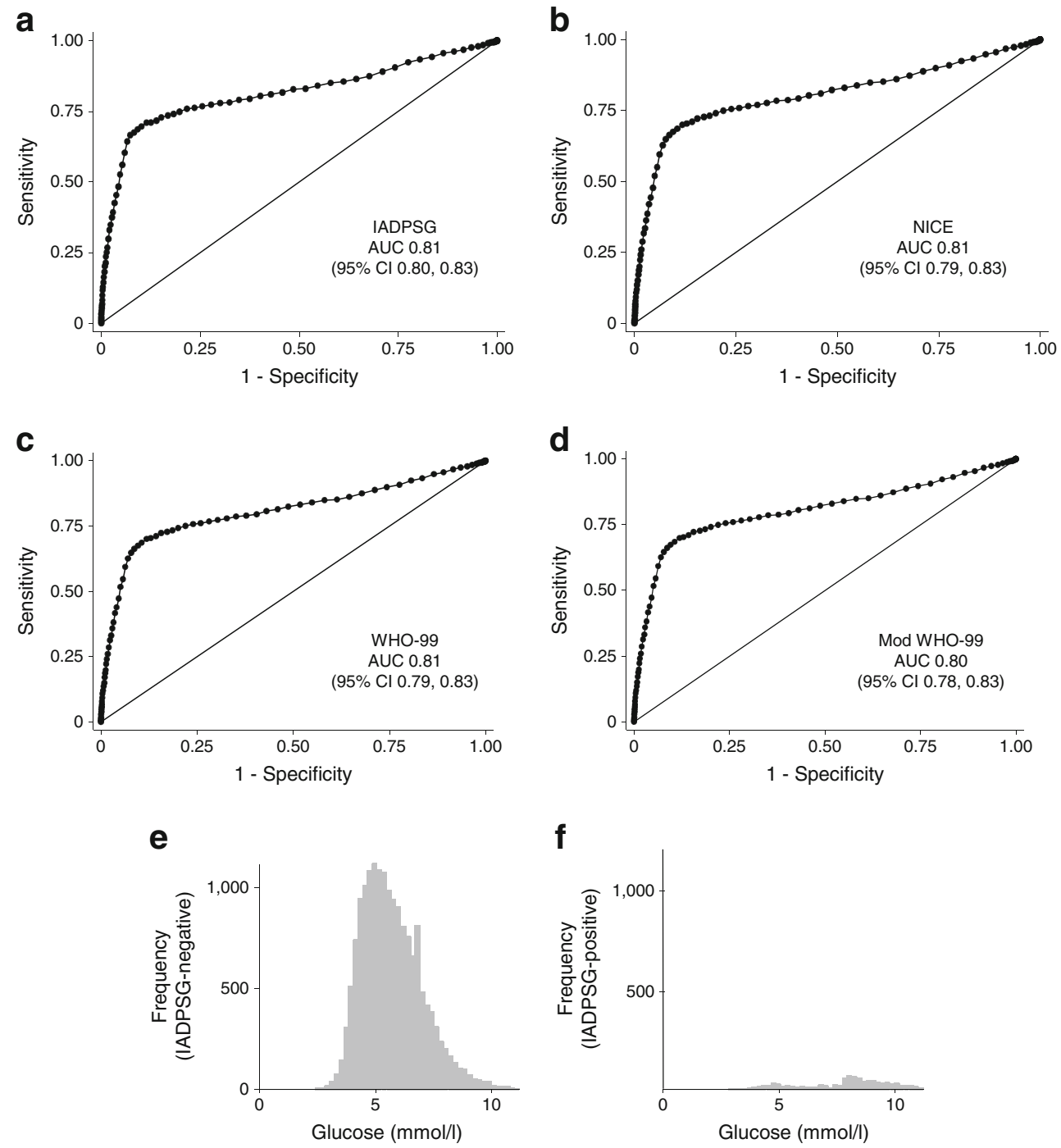

However, this low-risk group contained 269 women who were confirmed positive for GDM later in pregnancy $(30.4 \%$ of cases of GDM). Interestingly, our data suggests that these 269 women might not have been readily identified using risk-factor-based screening methods as approximately $38.7 \%$ were of normal pre-pregnancy BMI $\left(<25 \mathrm{~kg} / \mathrm{m}^{2}\right)$. They had a $33.7 \%$ risk of having an LGA infant (Fig. 2).

The use of RPG at booking compared favourably with other screening strategies in current clinical use (Fig. 3a, b). For example, maternal pre-pregnancy BMI and maternal age were both inferior at predicting GDM using the IADPSG criteria ( $n=1,181$ positive diagnoses, $n=884$ with RPG; BMI AUC 0.65, 95\% CI 0.63, 0.67; age AUC 0.60, 95\% CI 0.59, 0.62 ). Maternal age $\geq 30$ years predicted IADPSG GDM with a sensitivity of $74.8 \%$ and a specificity of $38.9 \%$. Maternal pre-pregnancy BMI $\geq 30 \mathrm{k} / \mathrm{m}^{2}$ predicted IADPSG GDM with a sensitivity of 0.30 and a specificity of 0.87 . Combining the risk factors age and BMI with RPG did not improve the overall predictive ability compared with using RPG alone
(Fig. 3c-f) when using thresholds of RPG $\geq 7.5 \mathrm{mmol} / \mathrm{l}$, age $\geq 30$ years and BMI $\geq 30 \mathrm{~kg} / \mathrm{m}^{2}$. However, combining age and BMI (Fig. 3c), RPG and age (Fig. 3d) or RPG and BMI (Fig. 3e) gave an improvement in test sensitivity to 0.83 0.95 , but at the cost of reducing the overall ROC AUC.

\section{Discussion}

This retrospective study in 17,736 pregnant women demonstrates that RPG at antenatal booking has reasonable performance as a screening test for GDM and performs better overall than screening based upon established risk factors (maternal age and BMI). RPG may have a role in identifying women who are at low risk of GDM and who would be considered to be of relatively low priority for early diagnosis or screening. Conversely, RPG could be used to identify women at high risk of GDM who might benefit from earlier diagnosis or from more intensive 
Fig. 2 Features of women with RPG above and below the $7.5 \mathrm{mmol} / 1(135 \mathrm{mg} / \mathrm{dl})$ cut-off point

Fig. 3 (a, b) ROC curves showing the use of maternal age at delivery (a) and pre-pregnancy BMI (b) for the prediction of GDM using the IADPSG criteria, as indicated in the graphs. $(\mathbf{c}-\mathbf{f})$ Combining the predictive value of RPG ( $\geq 7.5 \mathrm{mmol} / \mathrm{l})$, maternal age ( $\geq 30$ years) and maternal prepregnancy BMI $\left(\geq 30 \mathrm{~kg} / \mathrm{m}^{2}\right)$
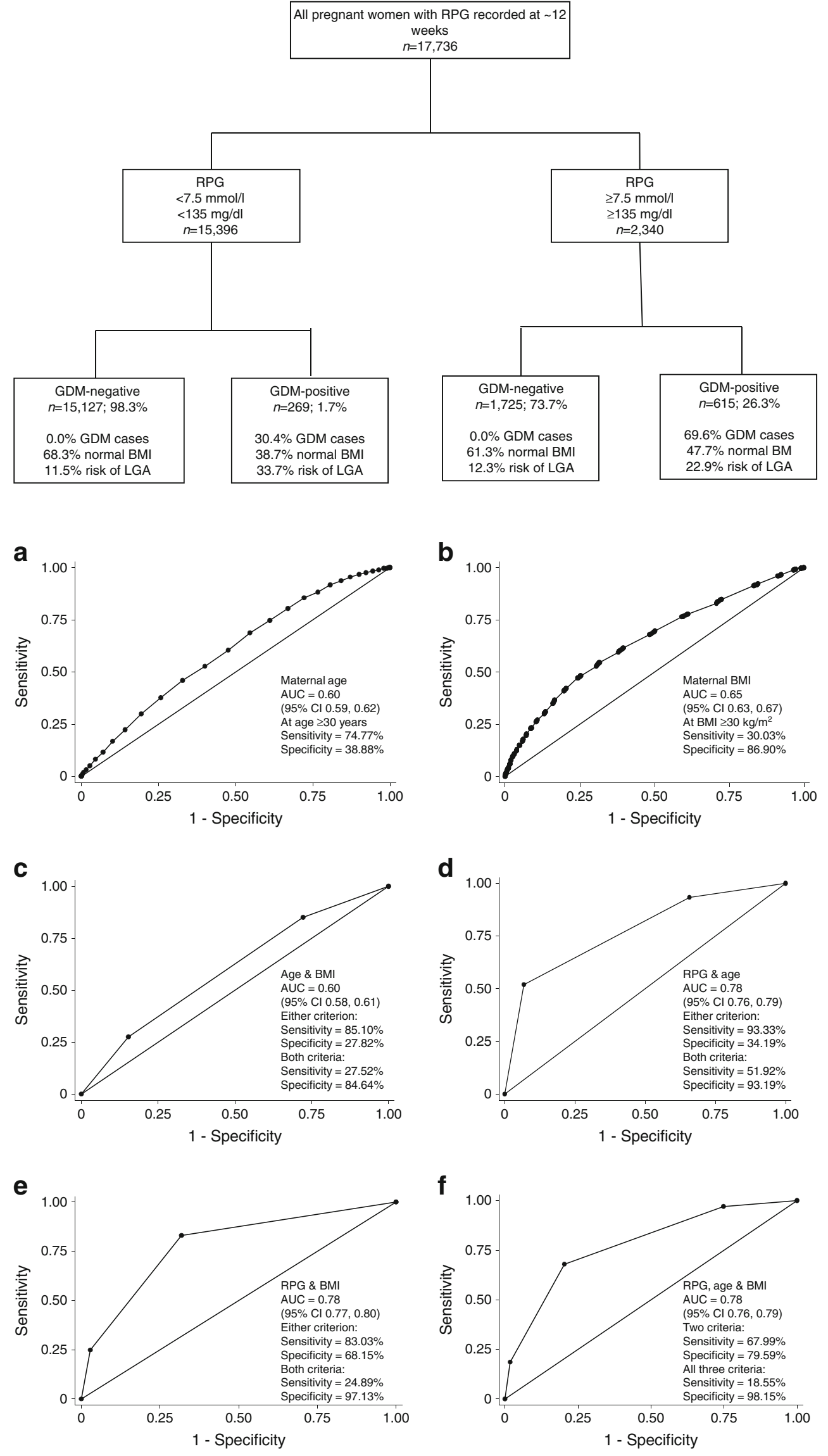
lifestyle interventions in early pregnancy. However, women with a low RPG still can develop GDM. This data suggests that reliance on the RPG at booking alone, without universal testing in the second trimester, would miss around $30 \%$ of cases of GDM. The test sensitivity could be improved by combining RPG with risk-factor information for age or BMI, but with a reduction in the specificity and overall ROC AUC.

This study has several strengths. First, the large sample size and unselected nature of the population allows robust assessment of the validity of RPG in clinical practice. Second, the RPG measured at antenatal booking was followed by universal screening for GDM using a twostep GCT and $75 \mathrm{~g}$ OGTT protocol. However, this protocol is no longer recommended by international guidelines $[4,5]$ and, importantly, GDM has not been definitively excluded using an OGTT in all 17,736 women. This was a single centre study in a relatively mono-ethnic population with a low prevalence of GDM. The overall performance of any screening tests may vary in different populations with different prevalence rates for GDM [16]. Over 7,000 women did not have evidence of an RPG measurement. These women have been described elsewhere and are otherwise comparable with the general population giving no evidence of selection bias [17]. The blood was analysed in a single accredited laboratory or using point-of-care devices under established laboratory quality control protocols. However, during this period both capillary and venous testing was used for glucose quantification in our institution. This may introduce small differences between measured and actual glucose concentrations. We have no data about the timing of OGTT testing in relation to the RPG although most OGTTs were performed at around 28 weeks following a GCT at 24-28 weeks. Although we have detailed information on some maternal risk factors, such as obesity and age, we do not have consistent information about previous history of GDM or family history of type 2 diabetes.

The use of RPG has many advantages. First, it is inexpensive and can be performed during the antenatal booking visit with no special pre-test preparation. Second, the opportunity to use point-of-care analysis on a capillary sample allows the clinician to have prompt access to the results, facilitating early lifestyle intervention or confirmatory testing. This may be particularly beneficial in resource-poor or rural environments where women travel a great distance to appointments. However, the RPG result is affected by pre-testing conditions, such as food intake and exercise, and this gives a wide range of RPG values in both GDM-positive and GDM-negative populations.

Previous studies of the RPG have had conflicting results. van Leeuwen and colleagues prospectively assessed the validity of RPG vs OGTT in 322 pregnant women and found that the RPG at 24-28 weeks had an ROC AUC of $0.69(95 \% \mathrm{CI}$ $0.61,0.78)[20]$. The same authors also performed a systematic review assessing the validity of RPG in the diagnosis of GDM. Six papers met the entry criteria and included data from the Netherlands [20], China [21], Japan [22], UK [15], India [23] and Kuwait [24], with a broad range of prevalence rates for GDM. All the studies dealt with the performance of RPG in the second or third trimester except for one study by Maegawa and colleagues [22] who studied 749 pregnant women in Japan (2.9\% had GDM). They found that the RPG and GCT had reasonable performance for detecting GDM in the first trimester. Some older studies indicated that GDM can be successfully diagnosed earlier in pregnancy [25-27], but the validity of using the WHO 2013 criteria outside the standard 24-28 week period has been questioned [28].

In our study, RPG with a sensitivity of $70 \%$ and specificity of $90 \%$ compares favourably with other approaches used for the screening of patients for GDM. A meta-analysis of the performance of the $50 \mathrm{~g} \mathrm{GCT}$, which included 13,564 women, showed that it had a sensitivity of 0.74 (95\% CI 0.62 , $0.87)$ and a specificity of $0.85(95 \%$ CI $0.80,0.91)$ for consecutive patients (not just those pre-selected based on risk factors) [29]. Other investigators have recommended a riskfactor-based approach. Göbl and colleagues designed a risk calculator based upon a woman's history of previous GDM, glycosuria, family history of diabetes, age, pre-conception dyslipidaemia and ethnic origin [14]. The risk calculator in addition to a fasting plasma glucose concentration was able to predict GDM with a ROC AUC of 0.9. However, in clinical practice, collecting detailed risk-factor information can be challenging and prone to error and pre-conception lipid results are often unavailable. Interestingly, other reports from a different ethnic population did not support the use of fasting blood glucose or risk-factor-based screening. Dahanayaka and colleagues in Sri Lanka found that fasting blood glucose alone was a poor predictor of GDM and that a risk-factorbased approach was only able to identify around two-thirds of affected women [12].

This study indicates that RPG measurement could form part of a useful testing strategy to identify women in early pregnancy who are at risk of developing GDM, or to prioritise second trimester OGTT testing to those most at risk of GDM. Interestingly, although GDM is thought to develop after 20 weeks' gestation in the majority of cases, this study shows that women in the first trimester can already be categorised biochemically according to their risk of later developing frank hyperglycaemia. In healthcare systems where universal biochemical screening with an OGTT is considered prohibitively expensive, RPG measurement at booking is likely to be a costeffective and convenient way of identifying women who need to be prioritised for early lifestyle intervention and an OGTT in the second trimester. 
Acknowledgements The authors acknowledge D. Church (Department of Clinical Biochemistry, Addenbrooke's Hospital), E. Te Braake, K. Stubbington (both from the Rosie Hospital, Addenbrooke's Hospital) and M. Wilson (Wolfson Diabetes and Endocrinology Clinic, Addenbrooke's Hospital) for their contributions to data collection and A. Thornton and A. Herrick (both from Information Technology Services, Addenbrooke's Hospital) for their contributions to data extraction.

Funding This project was not supported by any specific funding. CLM receives salary funding from the European Union Seventh Framework Programme (FP7/2007-2013; grant agreement no. 266408) and from the Wellcome Trust Translational Medicine and Therapeutics Programme, which is funded by the Wellcome Trust in association with Glaxo SmithKline.

Duality of interest CLM receives salary funding from the European Union Seventh Framework Programme (FP7/2007-2013; grant agreement no. 266408) and from the Wellcome Trust Translational Medicine and Therapeutics Programme, which is funded by the Wellcome Trust in association with Glaxo SmithKline.

HRM receives salary funding from an NIHR fellowship and has received honoraria for speaking engagements from Medtronic, Roche, Novo Nordisk and Eli-Lilly and is a member of the Medtronic European Advisory Board. DS has received honoraria from Astra Zeneca.

Contribution statement CLM designed the study, performed the data analysis and interpretation and wrote and revised the manuscript. HRM made a substantial contribution to data acquisition and analysis, reviewed and revised the manuscript and contributed to the discussion. DS identified the study questions, made a substantial contribution to data acquisition and analysis, reviewed and revised the manuscript and contributed to the discussion. All authors approved the final version of the manuscript prior to publication. The database used in this study has previously been used in other published works $[17,18]$.

CLM is the guarantor of this work and, as such, had full access to all the data in the study and takes responsibility for the integrity of the data and the accuracy of the data analysis.

Open Access This article is distributed under the terms of the Creative Commons Attribution 4.0 International License (http:// creativecommons.org/licenses/by/4.0/), which permits unrestricted use, distribution, and reproduction in any medium, provided you give appropriate credit to the original author(s) and the source, provide a link to the Creative Commons license, and indicate if changes were made.

\section{References}

1. Metzger BE, Coustan DR (1998) Summary and recommendations of the Fourth International Workshop-Conference on Gestational Diabetes Mellitus. The Organizing Committee. Diabetes Care 21(Suppl 2):B161-B167

2. Jensen DM, Korsholm L, Ovesen P, Beck-Nielsen H, MølstedPedersen L, Damm P (2008) Adverse pregnancy outcome in women with mild glucose intolerance: is there a clinically meaningful threshold value for glucose? Acta Obstet Gynecol Scand 87:59-62

3. Metzger BE, Lowe LP, Dyer AR et al (2008) Hyperglycemia and adverse pregnancy outcomes. N Engl J Med 358:1991-2002

4. Metzger BE, Gabbe SG, Persson B et al (2010) International Association of Diabetes and Pregnancy Study Groups recommendations on the diagnosis and classification of hyperglycemia in pregnancy. Diabetes Care 33:676-682
5. World Health Organization (2013) Diagnostic criteria and classification of hyperglycaemia first detected in pregnancy. Available from http://apps.who.int/iris/bitstream/10665/85975/1/WHO NMH_MND_13.2_eng.pdf, accessed 30 October 2015

6. American Diabetes Association (2014) Standards of medical care in diabetes - 2014. Diabetes Care 37(Suppl 1):S14-S80

7. Vandorsten JP, Dodson WC, Espeland MA et al (2013) NIH consensus development conference: diagnosing gestational diabetes mellitus. NIH Consens State Sci Statements 29:1-31

8. National Institute of Clinical Excellence (NICE) (2015) Diabetes in pregnancy: management of diabetes and its complications from preconception to the postnatal period. National Institute of Clinical Excellence (NICE) guideline NG3. Available from www. nice.org.uk/guidance/ng3/, accessed 30 October 2015

9. Cundy T, Ackermann E, Ryan EA (2014) Gestational diabetes: new criteria may triple the prevalence but effect on outcomes is unclear. BMJ 348:g1567

10. National Health Service. Gestational diabetes mellitus: screening, diagnosis and follow-up. Available from https://www.diabetes.org. uk/Documents/nhs-diabetes/pregnancy-preconception/gestationaldiabetes-screening-diagnosis-followup-national-overview.pdf, accessed 18 August 2015

11. Committee on Practice Bulletins (Obstetrics) (2013) Practice bulletin no. 137: gestational diabetes mellitus. Obstet Gynaecol 122: 406-416

12. Dahanayaka NJ, Agampodi SB, Ranasinghe OR et al (2012) Inadequacy of the risk factor based approach to detect gestational diabetes mellitus. Ceylon Med J 57:5-9

13. Simmons D, Devers MC, Wolmarans L, Johnson E (2009) Difficulties in the use of risk factors to screen for gestational diabetes mellitus. Diabetes Care 32, e8

14. Göbl CS, Bozkurt L, Rivic P et al (2012) A two-step screening algorithm including fasting plasma glucose measurement and a risk estimation model is an accurate strategy for detecting gestational diabetes mellitus. Diabetologia 55:3173-3181

15. Jowett NI, Samanta AK, Burden AC (1987) Screening for diabetes in pregnancy: is a random blood glucose enough? Diabet Med 4: $160-163$

16. van Leeuwen M, Opmeer BC, Yilmaz Y, Limpens J, Serlie MJ, Mol BW (2011) Accuracy of the random glucose test as screening test for gestational diabetes mellitus: a systematic review. Eur J Obstet Gynecol Reprod Biol 154:130-135

17. Church D, Halsall D, Meek C, Parker RA, Murphy HR, Simmons D (2011) Random blood glucose measurement at antenatal booking to screen for overt diabetes in pregnancy: a retrospective study. Diabetes Care 34:2217-2219

18. Meek CL, Lewis HB, Patient C, Murphy HR, Simmons D (2015) Diagnosis of gestational diabetes mellitus: falling through the net. Diabetologia 58:2003-2012

19. Kupke IR, Kather B, Zeugner S (1981) On the composition of capillary and venous blood serum. Clin Chim Acta 112:177-185

20. van Leeuwen M, Zweers EJ, Opmeer BC et al (2007) Comparison of accuracy measures of two screening tests for gestational diabetes mellitus. Diabetes Care 30:2779-2784

21. Tam WH, Rogers MS, Yip SK, Lau TK, Leung TY (2000) Which screening test is the best for gestational impaired glucose tolerance and gestational diabetes mellitus? Diabetes Care 23:1432

22. Maegawa Y, Sugiyama T, Kusaka H, Mitao M, Toyoda N (2003) Screening tests for gestational diabetes in Japan in the 1st and 2nd trimester of pregnancy. Diabetes Res Clin Pract 62: 47-53

23. Mathai M, Thomas TJ, Kuruvila S, Jairaj P (1994) Random plasma glucose and the glucose challenge test in pregnancy. Natl Med J India 7:160-162 
24. Nasrat AA, Johnstone FD, Hasan SA (1988) Is random plasma glucose an efficient screening test for abnormal glucose tolerance in pregnancy? Br J Obstet Gynaecol 95:855-860

25. Bartha JL, Martinez-Del-Fresno P, Comino-Delgado R (2000) Gestational diabetes mellitus diagnosed during early pregnancy. Am J Obstet Gynecol 182:346-350

26. Bartha JL, Martinez-Del-Fresno P, Comino-Delgado R (2003) Early diagnosis of gestational diabetes mellitus and prevention of diabetes-related complications. Eur J Obstet Gynecol Reprod Biol 109:41-44
27. Super DM, Edelberg SC, Philipson EH, Hertz RH, Kalhan SC (1991) Diagnosis of gestational diabetes in early pregnancy. Diabetes Care 14:288-294

28. Zhu WW, Yang HX, Wei YM et al (2013) Evaluation of the value of fasting plasma glucose in the first prenatal visit to diagnose gestational diabetes mellitus in China. Diabetes Care 36:586-590

29. van Leeuwen M, Louwerse MD, Opmeer BC et al (2012) Glucose challenge test for detecting gestational diabetes mellitus: a systematic review. BJOG 119:393-401 